\title{
Variability Modeling and Product Derivation in E-Business Process Families
}

\author{
Arnd Schnieders and Frank Puhlmann \\ Business Process Technology Group \\ Hasso-Plattner-Institute for IT Systems Engineering at the University of Potsdam \\ D-14482 Potsdam, Germany \\ \{arnd.schnieders, frank.puhlmann\}@hpi.uni-potsdam.de
}

\begin{abstract}
In this paper we present our findings in the area of process family architectures for e-business systems, described as variant-rich process models in the Business Process Modeling Notation (BPMN). We moreover address product derivation issues specific to process family engineering.
\end{abstract}

\subsection{Introduction}

Nowadays, process oriented software systems, like many business information systems, don't exist only in one single version, which covers the whole target market. Instead, many different variants of the system exist, which are specialized according to diverging customer needs. Until now, the corresponding customization has to be done manually, being a time-consuming and error-prone task. However, the ability to rapidly and cost-effectively develop and deploy customer tailored system variants is crucial to the competitiveness of companies developing business software. In order to cope with these challenges, techniques for the efficient production of similar software systems have been developed. These techniques, known as software product family engineering [1], have already been applied successfully in several enterprises [2]. However, up to now the investigation of product family engineering techniques for families of process oriented software, in short process family engineering, has been widely neglected. In this chapter we therefore present our findings in the area of process family architectures for e-business systems, described as variantrich process models in the Business Process Modeling Notation (BPMN) [3] as well as product derivation issues regarding the particularities in process family engineering.

This chapter is structured as follows: In section 1.2 we give a brief introduction to some basic concepts and describe in section 1.3 their application to an e-business process family. Section 1.4 introduces an approach for variability modeling in e-business process family architectures and for the automization 
and consistency maintenance during the derivation of process family members. In section 1.5 we illustrate our findings based on an exemplary process family of e-business shops. In section 1.6 we summarize the contents of this chapter.

\subsection{Preliminaries}

In this section we give a brief introduction to Process Family Engineering and Process Family Architectures.

\subsubsection{Process Family Engineering}

Product family engineering is a paradigm to develop software applications using a set of software subsystems and interfaces that form a common structure based on which derivative products tailored to individual customer needs can be efficiently developed [4]. Another important aspect is that within a software product family reuse isn't restricted to the reuse of implementation artifacts but is expanded to any development artifact (like e.g. requirement or design models).

Product family engineering is characterized by a so called dual lifecycle [5] as indicated in figure 1.1 [6]. In order to emphasize that our work focuses on the development of process-oriented software, we use the term process family engineering instead of product family engineering and process family infrastructure instead of product family infrastructure. However, the basic development process is the same for product family engineering as for process family engineering. In the first section of the process family development process (called process family engineering) generic development artifacts (called the process family infrastructure) are developed based on which process family members are derived efficiently in the corresponding phase within the second section (called application engineering) of the process family engineering process. During the derivation of process family members variation points containted in the generic artifacts of the process family infrastructure have to be configured according to the customer requirements. Thereby, dependencies between the variation points within the same as well as between different development artifacts have to be regarded. In our approach, we use an additional model, called variability management model, to control the dependencies between the variabilities within the process family. A specialty of process family engineering in contrast to product family engineering is that the process family architecture can act as a requirements artifact in addition to its role as design artifact. So, the customer can select a product not only based on the typical requirements artifact, like e.g. a feature model, but also based on the process family architecture. This has to be taken into consideration during product derivation. 


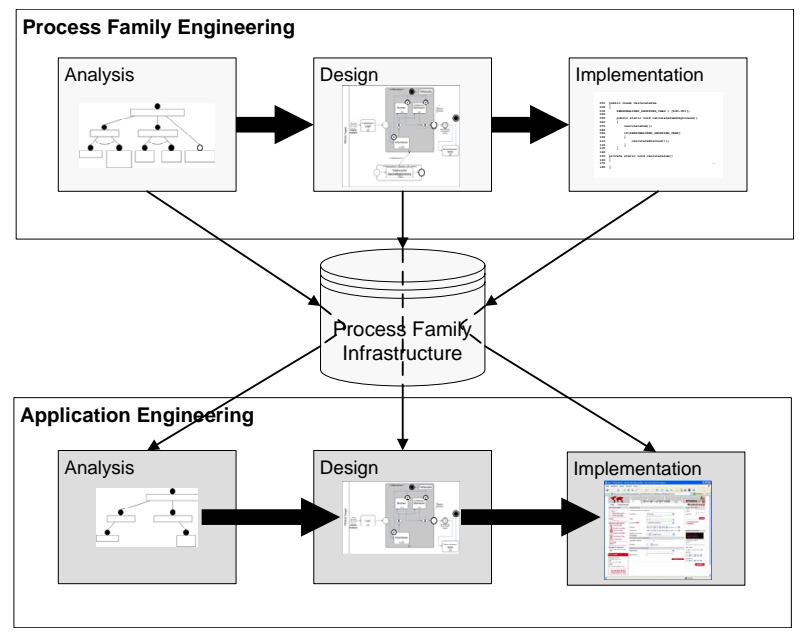

Figure 1.1. Process Family Engineering Process

\subsubsection{Process Family Architectures}

During the design of a process family a process family architecture (PFA) is developed based on the process family requirements. The PFA acts as reference architecture for the members of the process family and describes the basic structure for the applications of the process family. It defines the reusable system parts with their interfaces and covers both, the functional as well as the non-functional requirements on the process family. Moreover, the PFA describes which techniques shall be applied for realizing the variability (i.e. the variability mechanisms) and on which variation points they shall be applied. The selection of appropriate variability mechanisms is crucial for the design of the process family since they can have a substantial impact on the functional and non-functional properties of the system. Additionally, the proper selection of a variability mechanism guarantees for an easy generation of process family members based on the process family infrastructure.

Thus, for supporting process family engineering, concepts and a notation for process family architecture variability mechanisms (PFA variability mechanisms) are required, which allow for modeling architecturally relevant decisions concerning the realization of the system's variability. Later, during implementation, the process family architecture variability mechanisms are realized in the code by implementing variability mechanisms, which are both application domain as well as programming language dependent. Additionally, a binding time can be specified in the process family architecture for the variability, which is also application domain dependent. 


\subsection{E-Business Process Family}

In this section we describe how the concepts described in section 1.2 apply to the development of an e-business process family. We thereby follow the process family engineering reference model shown in figure 1.1.

Concerning the development of the process family infrastructure we follow the FODA feature diagram notation [7] to describe the requirements on the process family during the analysis phase. Based on these requirements a process family architecture is developed during the design phase using BPMN enhanced by the PFA variability mechanisms for variability modeling as described in the following section. Most of the variabilities within the process family architecture can be linked to an arbitrary combination of features in the feature diagram. However, there are also variabilities, which are specific to the process family architecture. In order to allow for an automatic product derivation as well as for maintenance reasons, the dependencies between the variabilities in the feature model and the process family architecture have to be maintained. Therefore, we introduce an additional model, the variability management model, which will be described in more detail in section 1.4. The variability management model also maintains the constraints between the variabilities within the feature model and the process family architecture. This makes sure that a consistent feature model and process architecture is derived during application engineering. In the implementation phase a program generator for the process family is implemented based on the process family architecture. However, here we won't go into detail concerning the generator implementation. More information can be found in [8]. Concerning the variability implementation, since we assume the e-business application to be written in Java, Java specific variability mechanisms are used to implement the PFA variability mechanisms applied in the BPMN based process family architecture. However, variability implementation issues will not be regarded in this chapter. More information about variability implementation can be found in [8].

In the analysis phase of application engineering the specific requirements on the e-business system to be developed are captured based on the feature model and the variant-rich BPMN model. During product derivation, the dependencies and constraints within the variability management model have to be regarded. The resulting application specific BPMN model is used as configuration model for the program generator. Based on the BPMN model the generator derives application specific Java components from the variant rich Java components and assembles them to the desired e-business system. 


\subsection{Modeling and Configuring Process Family Architectures in E-Business Process Families}

In this section we introduce an approach for modeling variability within BPMN models. Moreover, we deal with the problem of describing and maintaining the dependencies between the variabilities within the variant-rich BPMN model as well as between the feature model and the variant-rich BPMN model using a variability management model.

\subsubsection{Modeling Variability in BPMN}

According to the requirements for a process family architecture stated in section 1.2.2, a variant-rich business process diagram needs to contain three additions to standard business process diagrams. The first addition is a marking of the places where variability occurs (variation point). Second, the possible resolutions (variants) should be shown in the diagram. Third, the variability mechanism for realizing the variability should be represented. For providing the required additions to model variability within variant-rich BPMN models, we propose to adapt the concept of a stereotype from the UML2 specification to BPMN. Each BPMN activity, association, and artifact can have a stereotype attached.

The stereotype $\ll \operatorname{VarPoint} \gg$ is introduced to highlight variation points. The stereotype «VarPoint» can be further specialized. A «Abstract» variation point represents alternative behavior; it has to be resolved with a specific variant. $\mathrm{A} \ll N u l l \gg$ variation point represents optional behavior; it can be resolved with a specific variant. «Alternative» is a short representation of an abstract variation point with a specific variant which is the default resolution to this variation point. $\mathrm{A} \ll$ Optional» variation point is a short representation of a $\ll N u l l \gg$ variation point and a specific variant.

Variants are denoted using the stereotype $\ll$ Variant $\gg$. The $\ll$ Variant $\gg$ stereotype can also be expressed graphically as a puzzle-piece as a marker at the bottom of an activity. The stereotype «Variant» can have the tagged value Preconditions which provides information about the dependency of the subprocess variant from a certain feature and variant configuration. Since these dependencies can become arbitrarily complex, we provide only a simplified version in the process family architecture. If the selection of a subprocess variant requires the selection of a number of features and variants, the Preconditions tagged value will contain these features and variants as a list of comma-separated names. However, if the dependency is more complex, e.g. if a certain subprocess variant shall only be selected if feature 1 and 2 , and moreover variant 2 have been selected, but not feature 5 or 6 , the Preconditions tagged value would contain the following expression: F(feature 1, feature 2, variant 2, feature 5, feature 6). This indicates that the dependency is 


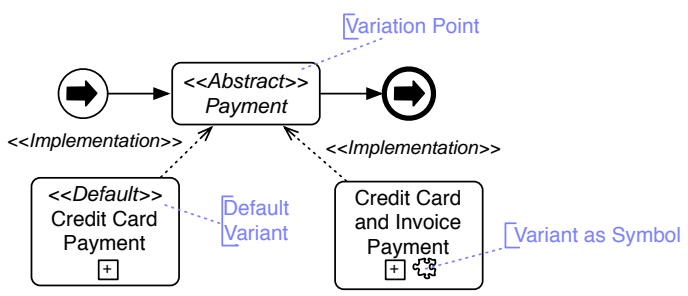

Figure 1.2. Encapsulation in BPMN.

a more complex one depending on the selection of the features and variants given as parameter values. The concrete dependency information is maintained within the variability management model. Additionally, the stereotype «Variant» can have the tagged value Effects providing information about the effects resulting from the selection of the variant. These are represented like the preconditions with the difference that no features, but only variants can be affected.

Variants are connected to their variation points by means of associations. The variability mechanism realizing the variability is assigned to the association as a third kind of stereotype. In general, variability mechanisms can be categorized into basic variability mechanisms and variability mechanisms, which are derived from the basic variability mechanisms. As the name indicates, basic variability mechanisms are stand-alone mechanisms, which don't require any other variability mechanisms. We have identified four types of basic variability mechanisms: encapsulation of varying sub-processes, parameterization, addition/omission/replacement of single elements, and data type variability. Since data flow is not supported directly in BPMN. Moreover, we support addition/omission/replacement of single elements only in a restricted form by BPMN inheritance. Concerning the second category of derived variability mechanisms we can further divide this category into variability mechanisms derived by restriction and by combination of other variability mechanisms. BPMN inheritance and extension are two examples for variability mechanisms derived by restriction and design patterns an example for a variability mechanism derived by combination. More details about BPMN variability mechanisms can be found in [8].

For a more convenient illustration, the stereotype «Variable» can be used to denote variability below the level of detail currently shown. Figure 1.2 gives an example for modeling a variation point and two associated variants using the variability mechanisms encapsulation of varying subprocesses in this case. 


\subsubsection{Variability Management for Automatic and Consistent Product Derivation}

The variability management model allows for a consistent and partly automatic configuration of the feature model and the variant-rich BPMN model. In this chapter, we will focus on the dependencies related to the feature model and variant-rich process model, while we do not consider implementation artifacts.

The variability management model contains of a set of links to the variation points of every variant-rich process family artifact. Moreover, for every variation point the variability model maintains references to the corresponding variants. For every variant an internal identifier as well as the preconditions and effects for the selection of the variant are stored. The preconditions and effects of a variant are represented in propositional logic. The internal identifier of a variant is used as literal for representing the variant in precondition and effect expressions. The precondition and effect expressions of the feature variants only contain literals that represent feature variants. The precondition expressions of the process model variants can contain literals representing both, feature as well as process model variants, while the effect expression can only contain literals representing process model variants.

The entirety of precondition and effect expressions within the variability model result in the process family constraints, which have to be fulfilled by any valid product configuration. So any product configuration, being represented as a set of selected feature model and process model variants has to fulfill the process family constraints. This has to be proved during product derivation. For verification, the selected and deselected feature and process model variants are represented as a set of expressions in propositional logic of the form variant $=$ true (in case the variant has been selected) and variant $=$ false (in case the variant has been deselected), while variant is the name of the literal representing the selected variant. So in order to verify that a configuration fulfills the process family constraints it has to be proved if the configuration fulfills the process family constraints. This can be accomplished by a SATchecker for example.

Moreover, if a variant has been selected a SAT-checker can calculate resulting configurations based on the process family constraints. The SAT-checker therefore checks whether new expressions of the form variant $=$ false or

variant $=$ true can be deduced from the process family constraints and the current configuration.

\subsection{Example}

This section presents a variant-rich process model for a process family of ebusiness shops also demonstrating some variability implementation details. A feature model is given in figure 1.3. An e-business shop consists of three 


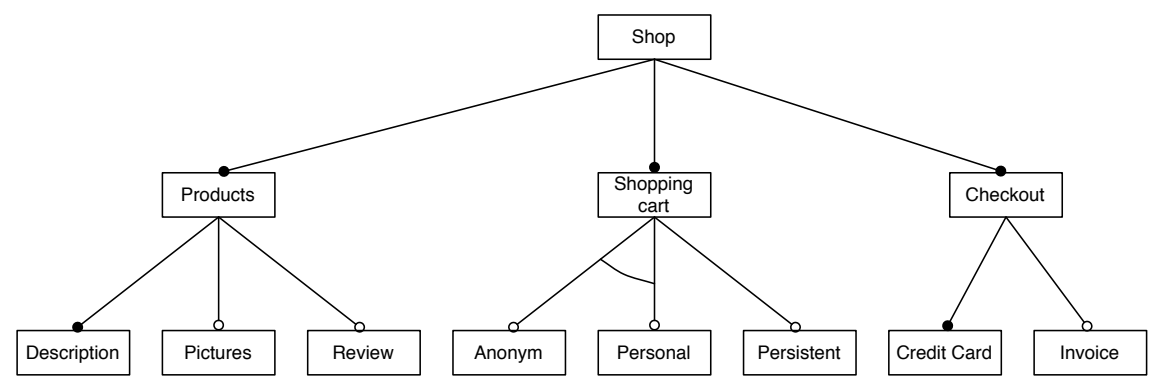

Figure 1.3. Features of the E-Business Shop.

mandatory features: products, shopping carts, and checkout. A product has a description as mandatory sub-feature, as well as optionally pictures and reviews. A shopping cart has three optional sub-features. It can either be anonymous (e.g. accessed by a proxy) or personalized. A personalized shopping cart allows for customer dependent discounts and for payment on invoice. Furthermore, a shopping cart can be made persistent, meaning that each time the customer returns, the shopping cart is loaded. The checkout has one mandatory sub-feature, offering a credit card checkout and an optional sub-feature invoice checkout.

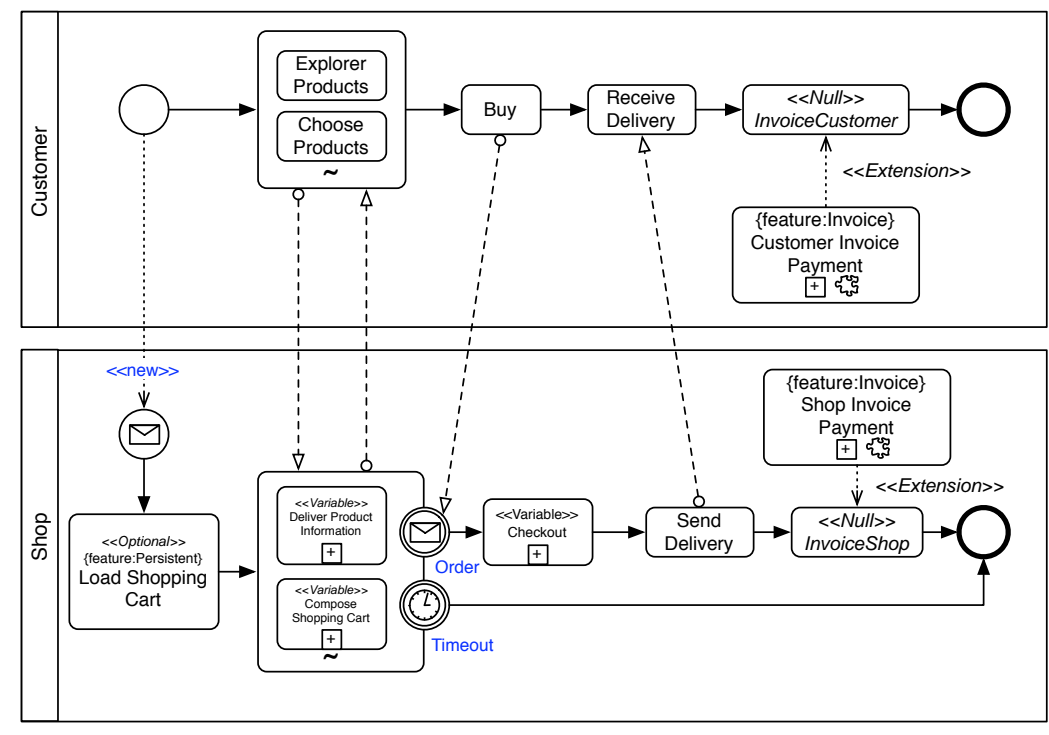

Figure 1.4. Top-level Process Model of the E-Business Shop. 
Figure 1.4 contains the variant-rich high-level process model of the ebusiness shop process family. We omitted the feature anonymous shopping cart for the sake of simplicity. A new instance of the shop's workflow is triggered by the customer starting a new browser instance, thereby also creating a new instance of the shop's process (denoted with $\ll$ New»). The customer then explores and chooses products in interaction with the shop, where the shop delivers product information and composes the shopping cart. If the customer decides to buy, she triggers the checkout sub-process of the shop. The shop then sends the delivery and both parties additionally handle invoice payment if the feature is included in this particular shop configuration.

The variant elements have been realized by different variability mechanisms. The customer's pool contains the optional behavior InvoiceCustomer, which is represented by a null activity. If the feature Invoice is selected, the sub-process Customer Invoice Payment is included at the extension point. The shop's pool has the optional task Load Shopping Cart, which is included if the feature Persistent (shopping cart) is selected. The null activity InvoiceShop is filled with Shop Invoice Payment if the invoice feature is selected. The task Shop Invoice Payment corresponds to the Customer Invoice Payment. The tasks Shop Invoice Payment and Customer Invoice Payment always appear together. The sub-processes Deliver Product Information, Compose Shopping Cart, and Checkout contain variability at a lower level, denoted with the «Variable» stereotype. For a lack of space we only consider the Checkout sub-process in detail.

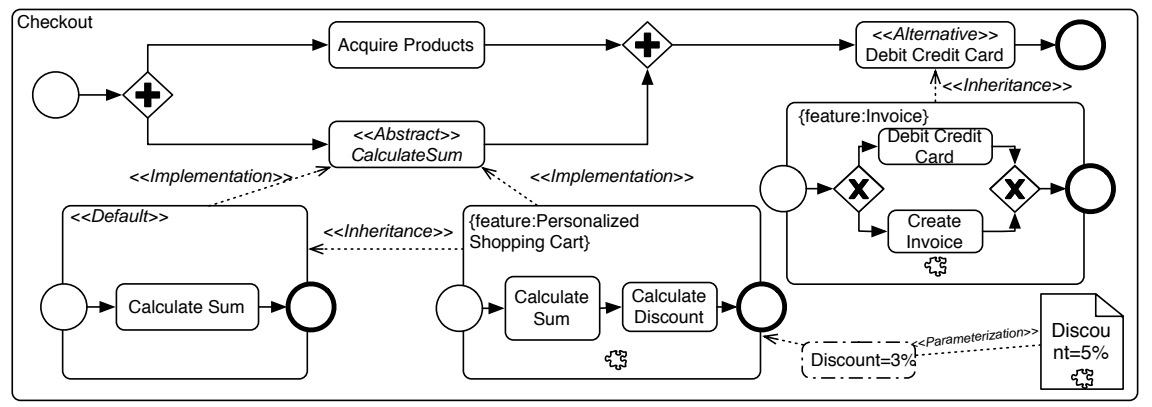

Figure 1.5. Checkout Sub-Process of the E-Business Shop.

Figure 1.5 expands the sub-process Checkout. It uses the concept of design patterns to describe the possible resolutions to the alternative variation point CalculateSum. The first resolution implements the default behavior, i.e. it only calculates the sum. The second, alternative resolution, specializes the default one by using inheritance to add the additional calculation of a discount. The percentage of the discount is parameterized with a default value of 3 . The 
task Debit Credit Card also has an alternative implementation derived by using inheritance.

Figure 1.6 shows an extract from the corresponding variability management model for the feature model and variant-rich process model. Optional features are represented by two variant identifiers. One represents the selected feature while the other one represents the deselected feature. In general all variants of the same variation point are mutually exclusive. This constraint hasn't been represented in the variability management model for the sake of readability. The constraints within the variability management model are formalized using propositional logic. Take for example variation point 3 $\left(P M_{-} V P 3\right)$ of the variant-rich process model. The corresponding constraint expression in propositional logic $\left(C \operatorname{Expr}\left(P M_{-} V P 3\right)\right)$ looks as follows:

$(($ ShopInvoicePayment $\wedge \neg N$ ull_VP3 $) \vee(\neg$ ShopInvoicePayment $\wedge$ Null_VP3) $) \wedge($ ShopInvoicePayment $\rightarrow$ Invoice $) \wedge($ ShopInvoicePayment $\rightarrow$ CreateInvoice $) \wedge($ ShopInvoicePayment $\rightarrow$ CustomerInvoicePayment $) \wedge$ $($ Null_VP3 $\rightarrow \neg$ Invoice $) \wedge($ Null_VP3 $\rightarrow \neg$ CreateInvoice $) \wedge($ Null_VP3 $\rightarrow$ $\neg$ Customer InvoicePayment)

The first part $\left(\left(\right.\right.$ ShopInvoicePayment $\left.\wedge \neg N u l l_{-} V P 3\right) \vee$ $(\neg$ ShopInvoicePayment $\wedge$ Null_VP3) $)$ makes sure that only one variant is selected for the variation point. The remaining expression reflects the preconditions and effects of the variant selection. Now, for getting a valid configuration of the process family the constraints for all variants have to be true. Thus the process family constraints look as follows: $C \operatorname{Expr}\left(F M_{-} V P 1\right) \wedge \ldots \wedge$ $C \operatorname{Expr}\left(F M_{-} V P 6\right) \wedge C \operatorname{Expr}\left(P M_{-} V P 1\right) \wedge \ldots \wedge C \operatorname{Expr}\left(P M_{-} V P 6\right)$

\begin{tabular}{|c|c|c|c|}
\hline $\begin{array}{l}\text { Variation } \\
\text { Point } \\
\text { Reference }\end{array}$ & Variant Identifier & Preconditions & Effects \\
\hline \multicolumn{4}{|c|}{ Feature Model } \\
\hline FM VP4 & Personal & & $\neg$ Anonym \\
\hline FM VP6 & Invoice & & Personal \\
\hline \\
\hline \multicolumn{4}{|c|}{ Variant-Rich Process Model } \\
\hline \multirow[t]{2}{*}{ PM_VP1 } & CustomerInvoicePayment & Invoice & $\begin{array}{l}\text { CreateInvoice } \wedge \\
\text { ShopInvoicePayment } \wedge\end{array}$ \\
\hline & Nul1_VP1 & $\neg$ Invoice & $\begin{array}{l}\neg \text { CreateInvoice } \wedge \\
\neg \text { ShopInvoicePayment } \wedge\end{array}$ \\
\hline \multirow[t]{2}{*}{ PM_VP3 } & ShopInvoicePayment & Invoice & $\begin{array}{l}\text { CreateInvoice } \wedge \\
\text { CustomerInvoicePayment }\end{array}$ \\
\hline & Null_VP3 & $\neg$ Invoice & $\begin{array}{l}\neg \text { CreateInvoice } \wedge \\
\neg \text { CustomerInvoicePayment }\end{array}$ \\
\hline \multirow[t]{2}{*}{ PM_VP4 } & CalculateSum & & \\
\hline & CalculateSumPersonalized & Personal & \\
\hline \multirow[t]{2}{*}{ PM_VP6 } & CreateInvoice & Invoice & $\begin{array}{l}\text { ShopInvoicePayment } \wedge \\
\text { CustomerInvoicePayment }\end{array}$ \\
\hline & DebitCreditCard & & $\begin{array}{l}\neg \text { ShopInvoicePayment } \wedge \\
\neg \text { CustomerInvoicePayment }\end{array}$ \\
\hline
\end{tabular}

Figure 1.6. Extract from Variability Management Model. 
Now let's assume that the customer wants an invoice to be created. Thus, in the variant-rich process model shown in figure 1.5 she selects the corresponding variant (the one derived from the Debit Credit Card activity using inheritance). This corresponds to the selection of the variant Create Invoice of the variation point $P M_{-} V P 3$ in the variability management model. Hence, CreateInvoice $=$ true. Now, the following follow-up configurations can be derived automatically based on the process family constraints:

\section{Variant-rich process model:}

- $\quad$ Invoice $=$ true, since CreateInvoice $\rightarrow$ Invoice $\left(\right.$ see CExpr $\left.\left(P M_{-} V P 6\right)\right)$

- ShopInvoicePayment = true, since CreateInvoice $\rightarrow$ ShopInvoicePayment (see CExpr $\left(P M_{-} V P 6\right)$ )

- CustomerInvoicePayment = true, since CreateInvoice $\rightarrow$ CustomerInvoicePayment (see CExpr(PM_VP6))

- DebitCreditCard = false, since CreateInvoice XOR DebitCreditCard (see $\left.C \operatorname{Expr}\left(P M_{-} V P 6\right)\right)$

- $\quad$ Null_VP1 $=$ false, since CustomerInvoicePayment $\rightarrow$ Null_VP1 (see $\left.C \operatorname{Expr}\left(P M_{-} V P 1\right)\right)$

- $\quad$ Null_VP3 = false, since ShopInvoicePayment XOR Null_VP3 (see $\left.C \operatorname{Expr}\left(P M_{-} V P 3\right)\right)$

\section{Feature model:}

- Personal $=$ true, since Invoice $\rightarrow$ Personal $($ see $C E x p r(F M-V P 6))$

- Anonym $=$ false, since Personal $\rightarrow \neg$ Anonym (see CExpr $\left(F M \_V P 4\right)$ )

In one of the last follow-up configuration steps Personal = true has been derived. Therefore, the precondition of the variant CalculateSumPersonalized of the process model variation point $P M_{-} V P 4$ has been fulfilled. A tool could now notify the user and ask him, whether CalculateSumPersonalized shall be selected.

\subsection{Conclusions}

Process Family Engineering hasn't been considered adequately in research so far. Therefore, in this chapter we have introduced an approach for process family architecture modeling and implementation, which contributes to a rapid and cost-effective development and deployment of customer tailored process-oriented business information system variants.

We have shown how a process family architecture for a family of e-business systems can be modeled in BPMN following a variability mechanism centric approach. We have also introduced an approach for the support of automated derivation of follow-up configurations during product derivation using a variability management model, which can economize the configuration work and assures the consistency of the resulting configuration. An algorithm for the automatic configuration would also prove user configurations before accepting 
them. Therefore, the satisfiability of the process family constraints with the current configuration has to be checked.

\section{References}

1. Becker, M.: Adaptation Support in Software Product Families (in German). PhD thesis, Technical University of Kaiserslautern (2004)

2. Reuys, A., Pohl, K., Gacek, C., Bermejo, J., Martínez, J.M., van der Sterren, W., Känsälä, K., Vehkomäki, T., Lerchundi, R., Martínez, R.A.C., Dueñas, J.C., Mittrach, S., Waeber, F., Berde, B., Sophie, V.: System Family Process Frameworks. ESAPS deliverable ESI-WP2-0002-04, University of Essen, Fraunhofer IESE, Sainco, Philips, Nokia, European Software Institute, Universidad Politécnica de Madrid, Siemens, Thomson-CSF/Alcatel LCAT (2000)

3. White, S.A.: Business Process Modeling Notation. BPMN 1.0, Business Process Modeling Initiative (2004)

4. Pohl, K., Böckle, G., van der Linden, F.: Software Product Line Engineering: Foundations, Principles, and Techniques. Springer (2005)

5. Weiss, D.M., Lai, C.T.R.: Software Product-Line Engineering: A Family-Based Software Development Process. Addison-Wesley Longman Publishing Co., Inc., Boston, MA, USA (1999)

6. European ESAPS Consortium: ITEA-ESAPS Full Project Proposal (1999)

7. Kang, K., Cohen, S., Hess, J.A., Novak, W.E., Peterson, A.S.: Feature-oriented domain analysis (foda) feasibility study. Technical Report CMU/SEI-90-TR-21 (1990)

8. Schnieders, A., Puhlmann, F.: Variability Mechanisms in E-Business Process Families. In Abramowicz, W., Mayr, H., eds.: Proceedings of the 9th International Conference on Business Information Systems (BIS 2006). Volume P-85, pp. 583601 of LNI., Bonn, Gesellschaft für Informatik (2006) 